\title{
sciendo
}

\author{
BULGARIAN ACADEMY OF SCIENCES
}

CYBERNETICS AND INFORMATION TECHNOLOGIES • Volume 19, No 1

Sofia 2019 Print ISSN: 1311-9702; Online ISSN: 1314-4081

DOI: $10.2478 /$ cait-2019-0007

\section{Multi-Channel Target Shadow Detection in GPS FSR}

\author{
Hristo Kabakchiev ${ }^{1}$, Ivan Garvanov ${ }^{2}$, Vera Behar ${ }^{3}$, Dorina \\ Kabakchieva ${ }^{4}$, Kalin Kabakchiev ${ }^{5}$, Hermann Rohling ${ }^{6}$, Krzysztof \\ $\mathrm{Kulpa}^{7}$, Alexander Yarovoy ${ }^{8}$
}

${ }^{1}$ Sofia University, 1164 Sofia, Bulgaria

${ }^{2}$ University of Library Studies and Information Technologies, 1784 Sofia, Bulgaria

${ }^{3}$ Institute of Information and Communication Technologies, 1113 Sofia, Bulgaria

${ }^{4}$ University of World and National Economy, 1700 Sofia, Bulgaria

${ }^{5}$ University of Birmingham, B15 2TT Birmingham, United Kingdom

${ }^{6}$ Technical University of Hamburg-Harburg, 21073 Hamburg, Germany

${ }^{7}$ Technical University, 00-665 Warsaw, Poland

${ }^{8}$ Technical University, 2600 Delft, Netherland

E-mails:ckabakchiev@fmi.uni-sofia.bgi.garvanov@unibit.bg behar@bas.bgrd.kabakchieva@unwe.bg kalin.kabakchiev@gmail.com rolingr@tu-harburg.dekkulpa@ise.pw.edu.pla.yarovoy@irctr.tudelft.nl

\begin{abstract}
The paper offers new application of a Multi-channel Forward Scatter Radar (MFSR), which uses GPS signals for detection of air targets on their GPS radio shadows. The multi-channel GPS MFSR detector consists of several channels, which process information from several satellites simultaneously. The phenomena of diffraction in the near area is used for shadow target detection. The target is considered to be detected, if it is detected at least in one of detector channels. Two experiments have been made to verify the proposed detection algorithm. The results obtained show that the proposed multi-channel detection algorithm can be successfully used for detection of low-flying air targets at very short distances or the near area of diffraction. Such targets are undetectable in GPS bistatic radar.
\end{abstract}

Keywords: FSR, GPS, signal detection and estimation.

\section{Introduction}

In bistatic radar, one of the factors affecting the signal strength at the radar receiver is the angle that the target makes to the transmitter and receiver, called the bistatic angle $\beta$. When the bistatic angle is equal or near $180^{\circ}\left(\beta \approx 180^{\circ}\right)$, the radar system is referred to as a Forward Scatter Radar (FSR) system [1]. The FSR technology exploits the phenomenon of diffraction of electromagnetic waves in order to detect targets. The diffraction is observed when the wavelength of electromagnetic waves, incident on the target, is much less than the size of the target. Especially, the FSR technology actively exploits the diffraction of the transmitted electromagnetic waves 116 
in the far zone of Faunhofer, when the target moves near the baseline "receivertransmitter" and far from the receiver. In that case the Forward Scatter (FS) effect is observed, the most attractive feature of which is the drastic increase in the target Radar Cross Section (RCS), and therefore, the strong increase of Signal-to-Noise Ratio (SNR) of the received signal. In case of the FS effect, the target partially blocks the signal wave front from the transmitter. According to the EM field theory and Babinet's principle given in [1], when there is an absolutely black body that is placed in the path of wave propagation and the dimensions of this body are large compared with the wavelength, then a scattered field exists behind the body ("shadow" "field"). This target shadow is an electromagnetic field, which is blocked by a target. In that case the target RCS depends only on the target silhouette area and wavelength of the electromagnetic field. In practice, the FS effect can occur in two cases. In the first case, when the moving target crosses the virtual baseline "transmitter-receiver", the target forward scatter RCS depends on the area of the side silhouette of the target. In the second case, when the target moves near and in parallel to the baseline "transmitter-receiver", the target forward scatter RCS depends on the area of the frontal silhouette of the target. The FSR systems have some limitations - lack of resolution in distance, the target must be located very close to the baseline "transmitter-receiver" in order to be detected.

Over the past decade, a new direction has emerged in radar research called Secondary Application of Wireless Technology (SAWT). The essence of this technology is to realize all possible radar applications on the base of wireless communications and radio navigation networks. One of these applications is to use satellites of the GPS navigation system for target detection in a radar system. For example, the use of passive radar systems where GPS satellites are used as transmitters are becoming increasingly popular as an alternative to traditional radar systems. The GPS Forward Scatter Radar (GPS FSR) is a specific case of FSR, where GPS satellites are exploited as "transmitters of opportunity". In [2, 3], authors consider the possibility to detect air targets in bistatic and forward scatter radar systems, which exploit GPS satellites as transmitters. These articles offer the theoretical analysis of possibilities to detect air targets [2] or sea targets [3] in bistatic radar systems, and also consider the difficulties in the practical implementation of such systems. In [2], the main difficulty is related to the separation of the direct satellite signal from the signal scattered from the target, when the target is located close to the base line "receiver-satellite". In [3], the exploitation of GPS signals backscattered from sea targets is proposed to enhance the target return with respect to the sea clutter. The idea to apply a GPS L1 receiver to FSR for detection of air targets is discussed in [4]. Some experimental results for air target detection using GPS L1-based FSR system are presented and discussed in [5]. In this article, authors describe the experiment made with a FSR system that detects an aircraft when it is moving parallel to the runway for landing and takeoff at the airport, i.e., in the case of a frontal forward scattering cross-section of the airplane. Authors consider the case when the aircraft is located in the area of deep (geometric) shadow, i.e., the signal scattered from the aircraft is blocking the signal from satellites of GPS. A possible algorithm for air target detection using GPS L5-based FSR system is described in [7], 
and the detection probability characteristics are analytically calculated in [8] for lowflying and poorly maneuverable air targets in the urban interference environment. In these two articles authors have discussed the potential to increase SNR for detection of aircrafts with GPS L5-based FSR system, which are due to the increase in radiated power of GPS L5 signal and the maximum value of a forward scattering cross-section of the airplanes being tested in the article. The next few articles [7,9] are devoted to experimental measurements made by using GPS L1-based FSR system and the Software-Defined GPS receiver [6], developed by the Aerospace Department in the University of Colorado, allowing to observe the geometric shadows (signal blocking) of ground objects of different sizes, mobile and stationary. The radio shadows of targets are formed by means of navigation messages at the output of Code \& Carrier block of the Software-Defined GPS receiver. Next, the navigation messages are integrated within hundreds of milliseconds in order to form the radio shadow of the object and the integrated messages are used for further detection of the object on his radio shadow. The absence of a priori information about the constellation of satellites in a given place and time also complicates to conduct planned experiments. In these experiments, the choice of satellites, at which is observed the deepest shadow from the object, estimation of the type and parameters of radio shadows are carried out by the operator, not automated. The purpose of these experiments was to clarify the real possibilities of the proposed system for recording radio shadows created by different objects - depending on the object's size, distance from the receiver to objects, speed of objects, and satellite constellation at the time of recording. After a series of experiments conducted at different times and conditions, we have found the opportunity to record radio shadows of different objects, such as buildings, bridges, cars, buses, and pedestrians.

In our previous article on GPS-based FSR [7], we consider the possibility of building a single-channel GPS-based FSR system. That means that in this case we choose only that satellite, which is visible and to the most extent satisfies the conditions for the appearance of the FS effect, i.e., which has the lowest elevation and its location is closest to the baseline "satellite-GPS receiver". This satellite ensures the best detection of radio shadows created by objects of various types, terrestrial and aerial. The concept and structure of multi-channel GPS navigation processing allows it to present the processing in the multi-channel GPS FSR system in the terms of multi-channel radar processing. In such a multi-channel GPS-based FSR system, one and the same target is irradiated by several satellites that satisfy the conditions of the FS effect (Fig. 1). This enables us to use in the multi-channel GPSbased FSR system the multi-channel signal processing approach, well-known in the radar technology. The advantage of this multi-channel approach to signal processing is that, depending on the type of a multi-channel system (decentralized or centralized) and the processing algorithms used, it is possible more or less to improve the detection characteristics.

In this article, we suggest to carry out a multi-channel detection of signals from different satellites irradiating one and the same target at the same time, like in a decentralized radar system. In our previous articles, we have described the signal processing in a single-channel GPS-based FSR system [7-10]. Our hypothesis about 
the ability to form the FS effect, maybe weaker in several other bistatic systems "target satellite", remains valid. This is based on the many experimental results obtained in our studies using a single-channel GPS-based FSR system for detection of various types of moving targets, ground and aerial [7-10]. The novelty is in the proposed multi-channel algorithm for detecting GPS signals from targets, which simultaneously processes GPS signals with different codes, performs CFAR detection in each channel of the respective GPS signal, and after that performs a secondary detection of a sequence of zeros and units according to the K/M criterion, or another criterion. This inter-channel processing allows you to decrease the false alarm probability and increase the probability of target detection. The proposed multichannel GPS shadow detector enhances the protection quality of a particular security zone, and independent of the type of moving targets, ground and aerial. This article demonstrates the work of the multi-channel GPS detector using the experimental recordings of signals obtained in 2017 during the observation of the take-off and landing airplanes at Sofia Airport.

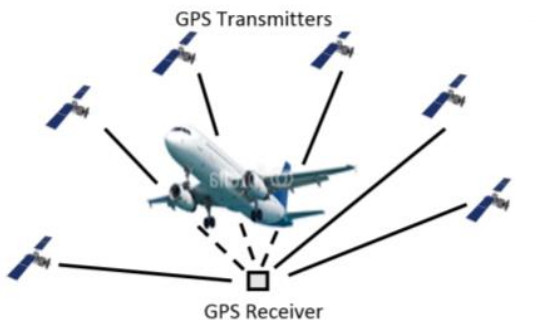

(a)

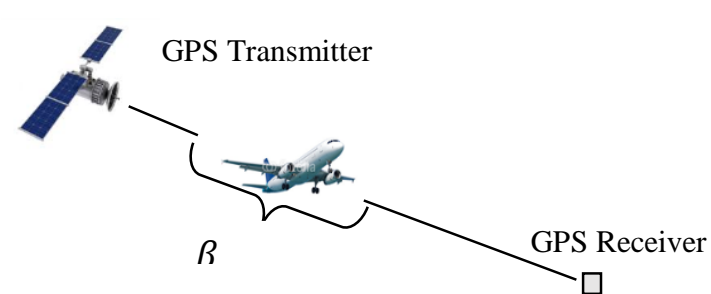

(b)

Fig. 1. GPS FSR: Multi-channel (a), single-channel (b)

In our experiments, we used the conventional GPS front-end and one commercial omnidirectional antenna with a small gain in several decibels. The airplanes were landing and taking off at the airport-Sofia and they were at low altitudes. The obtained results show that if we have the appropriate professional antenna, amplifier and front-end, then the airplanes can be detected at much greater distances by the proposed GPS-based MFSR when using their radio shadows in the near zone of diffraction.

\section{Target radio shadow formation}

If the distance from the receiver to the target $(R)$ is comparable to the size of the object $(D)$, then the target creates the "geometric shadow" of electromagnetic waves incident on the target. In the zone of "geometric shadow" act the laws of geometrical optics, i.e., electromagnetic waves spread straightforward. In that zone the distance to the target meets the inequality $R<<D^{2} / \lambda$ where $\lambda$ is the wavelength. In the near diffraction zone, i.e., Fresnel zone, the diffracted electromagnetic waves mutually interfere, and for the distance from the receiver to the target is valid the approximate equality $R \leq D^{2} / \lambda$. In the far zone of diffraction, i.e., Fraunhofer zone, the distances from the transmitter to the target and from the target to the receiver are much larger 
than the size of the target. In this area is valid the inequality $R>>D^{2} / \lambda$. For example, the diffraction of light passed through the circular aperture is shown in Fig. 2.

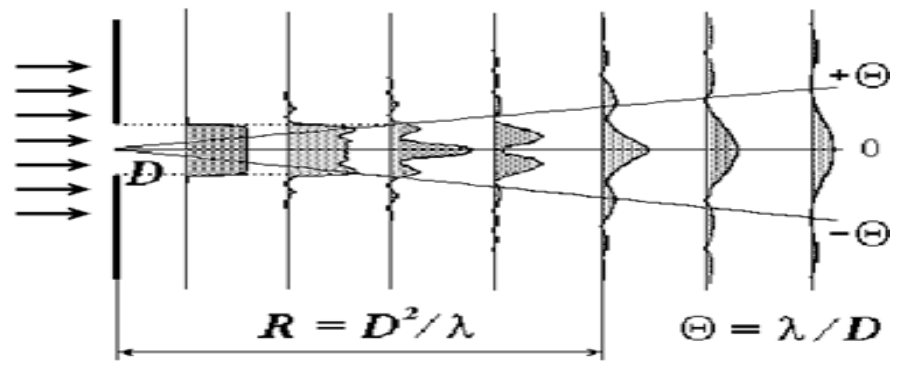

Fig. 2. Diffraction of light passed through the circular aperture

The diffraction of light on the disc is shown in Fig. 3. According to the Babinet's principle, the diffracted signal in this case, only changes the sign compared to the diffracted signal passed through the circular aperture.

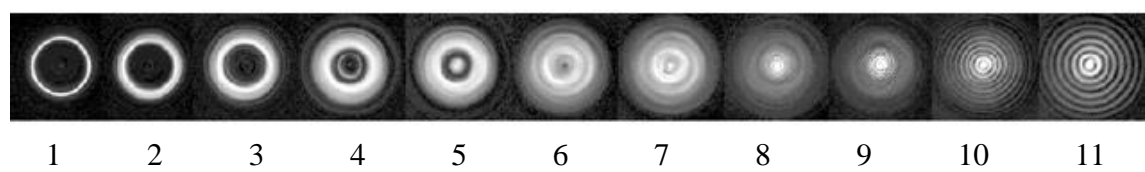

Fig. 3. Diffraction of light on the disc

(1,2 - zone of geometric shadow; $3 \div 7$ - Fresnel zone; $8 \div 11$ - Fraunhofer zone)

The FSR technology actively exploits the diffraction of the transmitted electromagnetic waves in the far zone of Fraunhofer when the target moves near the baseline "receiver-transmitter" and far from the receiver. In that case the Forward Scatter (FS) effect is observed, the most attractive feature of which is the drastic increase in the forward scattering radar cross-section, and, therefore, the strong increase of Signal-to-Noise Ratio (SNR) of the received signal.

\section{Power budget of a single-channel GPS FSR}

In case of bistatic passive radar that uses signals from GPS satellites, the basic radar equation can be written as [2]

$$
\mathrm{SNR}=\frac{S_{\text {direct }} \lambda^{2} G_{r} \sigma_{0}}{(4 \pi)^{2} R^{2} N_{r}} G_{\mathrm{SP}} G_{\mathrm{f}}
$$

where SNR is the SNR at the detector input, $\sigma_{0}$ is the target RCS, $R$ is the distance to the target, $S_{\text {direct }}$ is the power density of the transmitted signal prior to being reflected by the target $\left(\mathrm{W} / \mathrm{m}^{2}\right), N_{\mathrm{r}}$ is the thermal noise at the room temperature of $290 \mathrm{~K}\left(N_{\mathrm{r}}=-141 \mathrm{dBw}.\right), G_{\mathrm{SP}}$ is the processing gain at the output of IF signal processing and $G_{\mathrm{f}}$ is the improvement in SNR at the Moving Average Filter (MAF) output. The null-to-null bandwidth of the main lobe of the spectrum of L1 C/A code is $\Delta f=2.046 \mathrm{MHz}$ and the $\mathrm{C} / \mathrm{A}$ code length is $T_{\mathrm{s}}=1 \mathrm{~ms}$. Therefore the coherent processing gain is $G_{\mathrm{SP}}=10 \log 10\left(\Delta f \cdot T_{\mathrm{S}}\right)=33.1 \mathrm{~dB}$. 
In the far zone of diffraction where the FS effect appears when the target is located almost on the line "satellite-receiver" or when the bistatic angle is very close to $180^{\circ} \pm 2^{\circ}$, the target RCS strongly increases and depends only on the target silhouette area and the wavelength $\lambda$. In that case the target RCS can be calculated as [9]

$$
\sigma_{\mathrm{FS}}=\frac{4 \pi A_{\mathrm{tg}}^{2}}{\lambda^{2}}=\frac{4 \pi(h l)^{2}}{\lambda^{2}},
$$

where $A_{\mathrm{tg}}$ is the target silhouette area and the parameters $h$ and $l$ are geometrical dimensions of a target. In order to calculate the SNR at the CFAR detector input under conditions of the FS effect, the target RCS $\sigma_{0}$ in (8) must be replaced by (9). After substitution, the expression (8) takes the following form:

$$
\mathrm{SNR}_{\mathrm{FS}}=\frac{S_{\text {direct }} G_{\mathrm{r}}(h l)^{2}}{4 \pi R^{2} N_{\mathrm{r}}} G_{\mathrm{SP}} G_{\mathrm{f}} \text {. }
$$

\section{GPS multi-channel detector of a target shadow in the near zone of diffraction}

Here we use both a multi-channel approach and other specific techniques used in our previous studies to improve detection of air targets in intensive interference $[7,10]$. The aim of this article is to demonstrate the ability to automatically detect radio shadows created by low-flying airplanes at very short distance by using of a multichannel GPS L1-based FSR system, when there is no information which GPS satellites satisfy the conditions for the appearance of the radio shadow of airplanes. In our case, the multi-channel GPS FSR system can receive signals potentially from 32 GPS satellites (see Fig. 1a). In contrary to detection algorithms that use the navigation message for target detection discussed in [7, 9,10], we propose such a possible multi-channel algorithm for target shadow detection that is based on the direct processing of the GPS L1 signal received from satellites, and therefore without using the processing blocks of the Software-Defined GPS receiver. In our case, the multi-channel detector of a GPS FSR system can consist of 32 processing channels (Fig. 4).

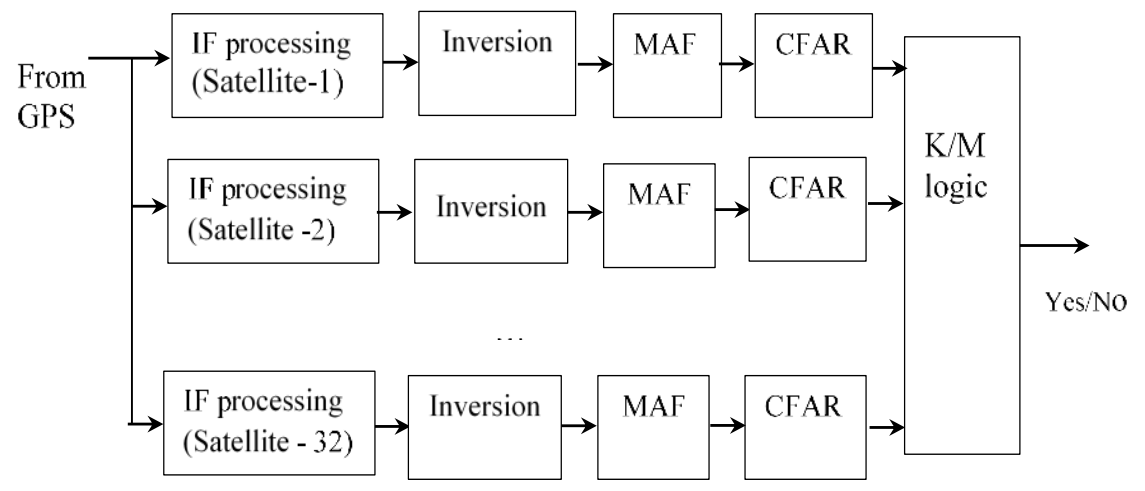

Fig. 4. General flow-chart of signal processing 
According to [1] we have possibility to increase the reliability of detection of targets in this detector by using the information obtained from all GPS satellites irradiating the same target. The general flow-chart of the proposed multi-channel algorithm for target detection is presented in Fig. 4. It is assumed that the incoming signal firstly is received by the RF front-end where it is down converted from RF frequency to an Intermediate Frequency (IF), digitized at a suitable sampling frequency and recorded as a digital file. As shown in Fig. 4, the algorithm for target detection is 32-channel, and each channel extracts and performs the signal from only one satellite irradiating the target. Under condition that it is unknown what satellites are visible at the moment, the signal processing is simultaneously carried out in 32 channels. The signal processing in each satellite channel includes two basic stages: the performance at an intermediate frequency (IF processing) and the performance at a low frequency (Inversion, MAF, CFAR). We propose to use the simplest algorithm to combine the information from all 32 processing channels, which is based on K/M logic.

\subsection{IF processing}

In each processing channel, the IF processing extracts the satellite signal reradiated by the target from the incoming data and forms a low-frequency signal for the further processing. As shown in Fig. 5, the extraction of a satellite signal is carried out through a multichannel matched filter in the frequency domain. Because the duration of the $\mathrm{C} / \mathrm{A}$ code is equals one millisecond, the incoming signal is divided by portions of $1 \mathrm{~ms}$, and processed sequentially portion by portion. In result of IF processing the low-frequency signal $Z$ is formed. In our case the formation of signal $Z$ in every processing channel is based on the power of the GPS signals in the frequency channels. However, there is the possibility to use also information about the phase GPS signals in order to form signal $Z$.

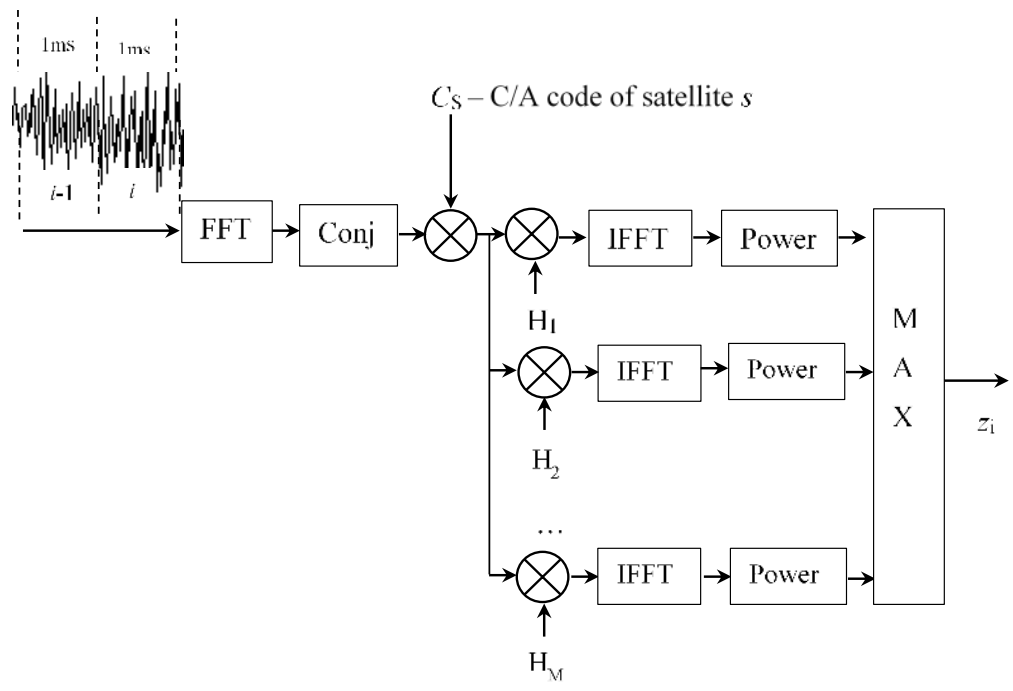

Fig. 5. Flow-chart of IF processing 
As shown in Fig. 5, the expected IF frequency range is divided into $M$ frequency channels $f_{m}, m=1, \ldots, M$, by step of $500 \mathrm{~Hz}$. This frequency range takes into account the expected Doppler frequency range of a high-speed aircraft and satellites. In each satellite channel, the output of the multichannel matched filter is formed as

$$
\begin{gathered}
y_{s, m}=\operatorname{IFFT}\left\{C_{s} \cdot H_{m} \cdot \operatorname{conj}(X)\right\}, \text { where } H_{m}=\exp \left(2 \pi j f_{m}\right), \\
m=1, \ldots, M, \text { and } s=1, \ldots, 32,
\end{gathered}
$$

where $C_{s}$ is the C/A code of satellite $s$, and $X$ is the spectrum of the incoming signal. The power of the output signal of the multi-channel matched filter is given by

(5) $\quad P_{s, m}=\operatorname{Re}\left\{y_{s, m}\right\}^{2}+\operatorname{Im}\left\{y_{s, m}\right\}^{2}$, where $m=1, \ldots, M, s=1, \ldots, 32$.

The sample $i$ of the low-frequency signal $Z$ is formed as the peak value of the power $P_{s, m}$ for all $m=1, \ldots, M$.

(6) $z_{s, i}=\max \left\{P_{s, m}, m=1, \ldots, M\right\}$ for each $i$-th 1 ms-portion, where $i=1, \ldots, I$, and the value of $I$ is the number of $1 \mathrm{~ms}$-portions of the incoming signal that is processed at the stage of the IF processing. In result of the IF processing the output signal $Z$ is sampled at the low frequency of $1 \mathrm{kHz}$. In our case, at the presence of the target radio shadow, the maximal of the signal power after matched filtering $\left(P_{s, m}\right)$ is near same in all frequency channels. Therefore, no matter in what frequency channel the maximal value of the signal power will form $z_{s, i}$.

\subsection{Inversion and filtration}

After the IF processing, the low-frequency signal $z$ is inverted, and the inverted signal takes the form

$$
q_{s}=\left|z_{s}-\max \left(z_{s}\right)\right|, s=1, \ldots, 32 .
$$

Next, the low-frequency signal is filtered by the Moving Average Filter (MAF) in order to improve SNR at the CFAR (Constant False Alarm Rate) detector input. The filter output is formed as

$$
q_{s, f}[n]=\frac{1}{2 N+1} \sum_{k=-N}^{N} q_{s}[n-k], s=1, \ldots, 32,
$$

where the parameter $2 N+1$ is the filter length. In order to speed the signal processing, the MAF can be replaced by the Moving Average Filter with a Jumping Window (MAFJW).

\subsection{CFAR detection}

In each processing channel, the CFAR signal detection is performed after signal filtration. The CFAR detection approach is based on the criterion of NeumannPearson. According to this criterion, the following algorithm can be used for testing a simple hypothesis $H_{1}$ (target is present) against a simple alternative $H_{0}$ (target is absent)

$$
\begin{aligned}
& H_{1} \text { if } \max \left\{q_{s, f}(n)\right\} \geq T_{\mathrm{fa}} \sum_{l=1}^{L} q_{s, f}(l), \\
& H_{0} \text { otherwise. }
\end{aligned}
$$

In that decision rule, $q_{s, f}(l)$ are the values of the filtered signal within the reference window of size $L$ needed for power noise estimation. The detection constant $T_{\mathrm{fa}}$ is determined in accordance with the probability of false alarm $P_{\mathrm{fa}}$, which should be 
maintained by the detection algorithm. In case of mean zero Gaussian noise, the detection constant can be calculated as

$$
T_{\mathrm{fa}}=P_{\mathrm{fa}}^{-1 / L}-1 .
$$

\subsection{K/M Logic}

For final target detection, we propose to use the simplest logical algorithm to combine the detections from all channels. It can be the logic " $K$ out of $M$ " where $M$ is the number of processing channels, and $K$ is the number of channels, in which the target was detected.

\section{GPS multi-channel detector of a target shadow in the far zone of diffraction}

The block-scheme of signal processing in the multi-channel algorithm for detection of target shadows in the far zone of diffraction when the FS effect appears is shown in Fig. 6.

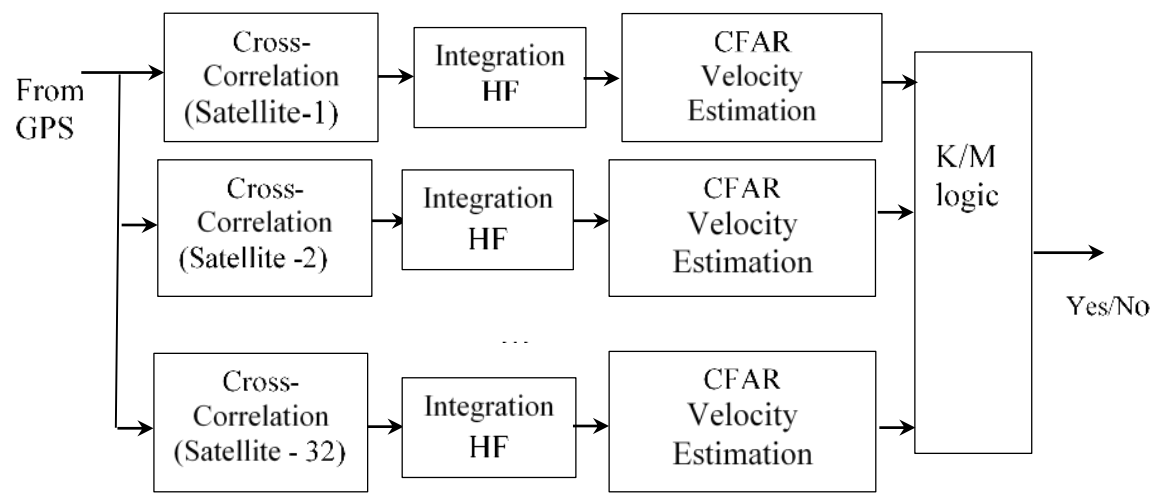

Fig. 6. Flow-chart of IF processing

In case of the SF effect, the incoming signal is a mixture of signals emitted by all visible satellites (direct signals) and those GPS signals, which are reradiated by a target moving nearly the baseline between GPS satellites and the GPS receiver (target signals). In the time domain, the direct signals are much more powerful than the signals reradiated by the target and mask them making the target impossible for detection. If the velocity of the target is large enough, then the Doppler frequency will also be quite large. In this case, the signal from the target can be separated from the direct signal by means of a high frequency filter.

\subsection{Cross-correlation}

The incoming signal $X$ is performed in the multi-channel cross-correlator, whose frequency responses in each satellite channel $(s)$ are products of the corresponding C/A code of a satellite $\left(C_{s}\right)$ and the high frequency function $\left(H_{0}\right)$ depending on the 
central frequency of the GPS L1 signal $\left(f_{0}\right)$. The output signal of the multi-channel cross-correlator is:

$$
y_{s}=\operatorname{IFFT}\left\{C_{s} \cdot H_{0} \cdot \operatorname{conj}(X)\right\}, \text { where } H_{0}=\exp \left(2 \pi j f_{0}\right), s=1, \ldots, 32 .
$$

\subsection{Coherent integration}

This procedure carries out a periodic integration of the input signal power during $N$ sequential repetition periods, each of them with the length of $1 \mathrm{~ms}$. When the number of such integrated periods grows, the output signal-to-noise ratio also grows with each integrated period.

\subsection{High frequency filter}

After cross-correlation, the spectrum of the direct signal from each satellite is centred at the zero frequency. However, the spectrum of the signal reradiated by the target is centred at its Doppler frequency. The purpose of HF filtering is to pass signals at the frequencies higher the cut-off frequency. The output signal is formed as convolution between the input signal and the impulse response of the filter. The cut-off frequency of the high frequency filter must be more than a half of the bandwidth of the GPS L1 signal.

\subsection{CFAR Detection and target velocity estimation}

In each satellite channel, the detection of target shadow is carried out in the frequency domain according to algorithm (9). When the CFAR detector indicates the detection of a signal in some satellite channel, then in this channel is carried out the estimation of the target velocity. The target velocity is evaluated through the estimate of the target Doppler frequency. The estimate of the target Doppler frequency is found as a frequency, at which the filtered power spectrum has the maximum value

$$
f_{d}=\operatorname{argmax}\left(P_{\text {filtered }}(f)\right) \text {. }
$$

Using (12), the estimate of the target velocity is computed as

$$
V=\frac{\lambda f_{d}}{2}
$$

\subsection{K/M Logic}

The rule "K out of M" is used to indicate the final target detection if several satellite channels register the target detection. The final velocity estimate is defined as a maximal estimate obtained in channels where the target signal is detected.

\section{Simulation results in the far zone of diffraction}

The results described in this Section are obtained using the computer stimulation approach. The signal processing algorithm, described in Section 5, is tested for one satellite channel after cross-correlation. The following parameters of the GPS L1 signal are used in simulation. They are: Receiver gain in $\mathrm{dB}$ is $G_{\mathrm{r}}=20$; transmitted 
power on the Earth in $\mathrm{dB}$ is $P_{\mathrm{t}}=-160$; GPS receiver noise in $\mathrm{dB}$ is $N_{\mathrm{r}}=-141$; number of integrated periods of the C/A code is $N=20$; coherent processing time is $P=0.001 \mathrm{~s}$; GPS receiver bandwidth is $B=2 \mathrm{MHz}$; wavelength $\lambda=0.19 \mathrm{~m}$; sampling frequency is $f_{0}=10 . \mathrm{B} \mathrm{Hz}$; pulse width is $W=4 / B \mathrm{~s}$, processing gain is $G \mathrm{sp}=10 \log 10(P \cdot B) \mathrm{dB}$.

The output signal of the cross-correlator is simulated as a sum of the three components: 1 - direct signal simulated as a Gaussian noise centred at the zero frequency; 2 - target signal centred at the target Doppler frequency and 3 - receiver noise simulated as zero mean Gaussian process with the noise power $N \mathrm{r}$. The simulation is carried out for a large spherical target with a diameter of $50 \mathrm{~m}$ that falls along of the baseline "GPS receiver-GPS satellite". As known, when the object moves along the baseline ,receiver-satellite" the FS effect appears at distances to the target more than $R_{\mathrm{FSR}}$. In this case the radar cross section of the meteoroid is calculated by (2).

The difference in SNR of the received direct signal and the re-radiated FS signal from the targets, after integration of 20 periods of C/A code, is shown in Fig. 7, depending on different target diameters $(D=10,30$ and $50 \mathrm{~m})$. It can be seen that this diffrence in SNR is directly proportional to the FS radar cross section (FS RCS) of the targets, and inversely proportional to the square of the distance to the object, and does not depend on the parameters of the GPS FSR system. These graphics are universal because they show the dependence of the difference in SNR between the direct and the re-radiated signal depending on the size of the falling targets and the distance to them, in FSR systems monitoring the space with different signal sources (satellites, pulsars, etc.). From the results in Fig. 7 it follows that the direct GPS signal received by the receiver on the surface of the Earth has a constant magnitude and it is represented as a straight line. Naturally, the magnitude of the direct signal depends on the number of coherent integrations of the signal at the output of the crosscorrelator.

It is impossible to detect the target signal in the time domain because it coincides in time with the direct signal. The power of the direct signal is much greater than that of the signal reradiated by the meteorite. The reradiated signal by the target has a high Doppler velocity of $20-40 \mathrm{~km} / \mathrm{s}$. Then the signal selection can be performed in the frequency domain. In this article we accept that satellite signals have the zero Doppler frequency. This assumption is plausible because the speed of GPS satellites is much smaller than that of targets. The one-sided spectrum of the direct signal and the signal from the target that moves at a speed of $20 \mathrm{~km} / \mathrm{s}$ is shown in Fig. 8 .

It follows from Fig. 8 that the spectra overlap and the detection of the target signal is difficult. If the target moves at velocity of $40 \mathrm{~km} / \mathrm{s}$, its spectrum is outside the spectrum of the direct signal (Fig. 9). To separate the two signals, we used a highfrequency filter that suppresses the direct and powerful GPS signal, and thus allows a target to be detected at speeds greater than $20 \mathrm{~km} / \mathrm{s}$. The result after filtration is shown in Fig. 10. The maximum frequency is $490 \mathrm{~Hz}$, which corresponds to $46.5 \mathrm{~km} / \mathrm{s}$. 


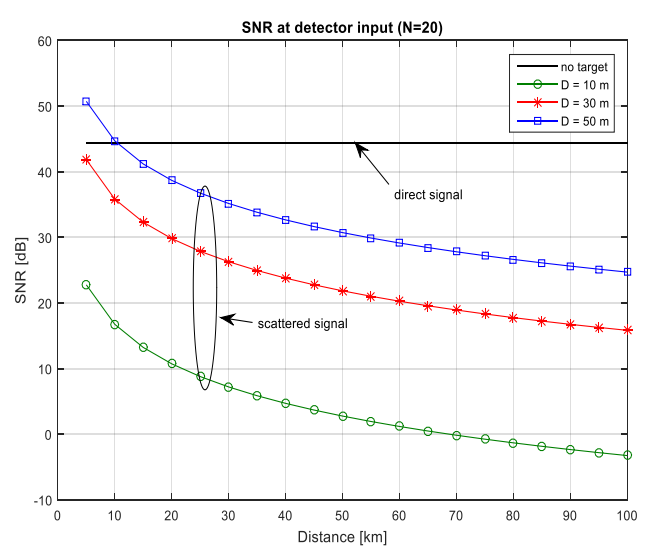

Fig. 7. SNR values of incoming signals

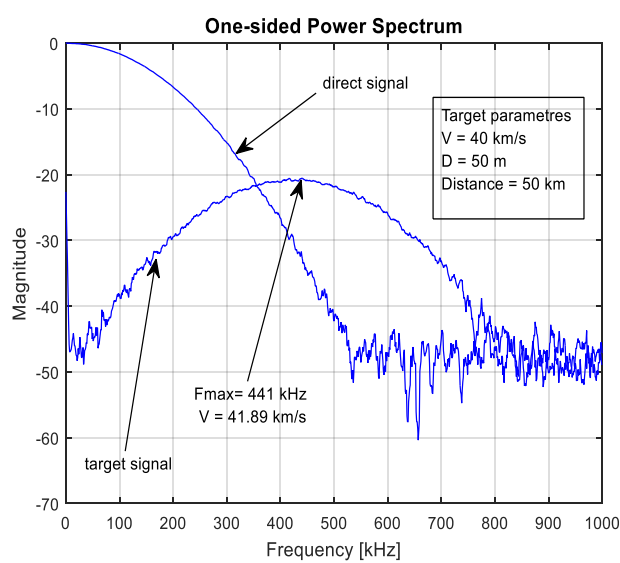

Fig. 9. One-sided spectra of signals $(V=40 \mathrm{~km} / \mathrm{s})$

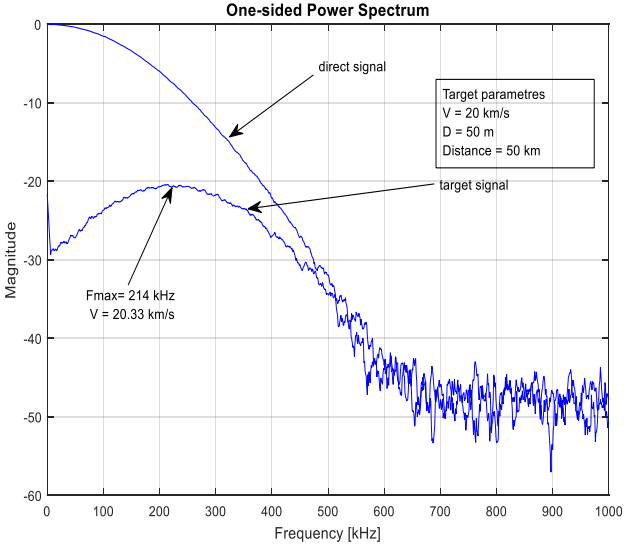

Fig. 8. One-sided spectra of signals $(V=20 \mathrm{~km} / \mathrm{s})$

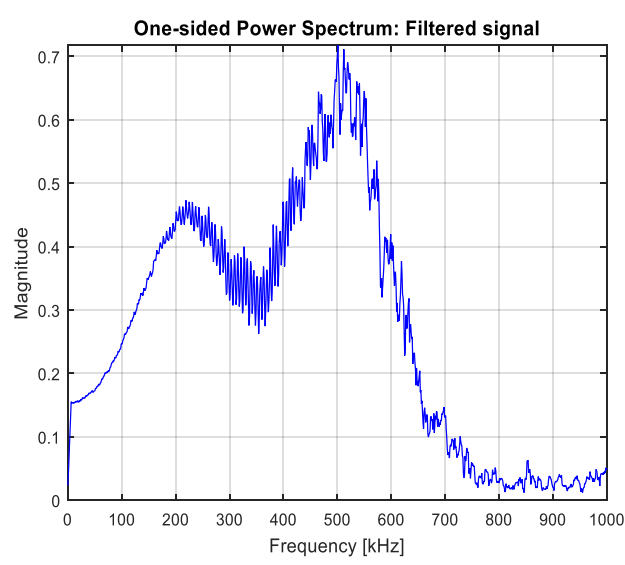

Fig. 10. One-sided spectrum at the HF output

\section{Experiment description and results in the near zone of diffraction}

In our experiments we use the GPS recording system GNSS_SDR, which is proposed and developed in the Aerospace Department of the Colorado University, USA [6]. This recording system receives and records the raw GPS data flow using the small commercial GPS antenna and the USB-based device (Fig. 11 right). The recorded GPS signals are saved as binary files in the computer memory. The second device used in our experiments is the GPS receiver (Antaris AEK-4R), which we used to see the location of the satellites over the horizon (Fig. 11, left).

During the experiment, the position of all visible satellites found by the GPS receiver "Antaris AEK-4R" is shown in Fig. 12. At the center of the image is the position of our GPS receiver during the experiment. The strength of the GPS signal 
acquired from all visible satellites by the GPS receiver "Antaris AEK-4R" is shown in Fig. 13.

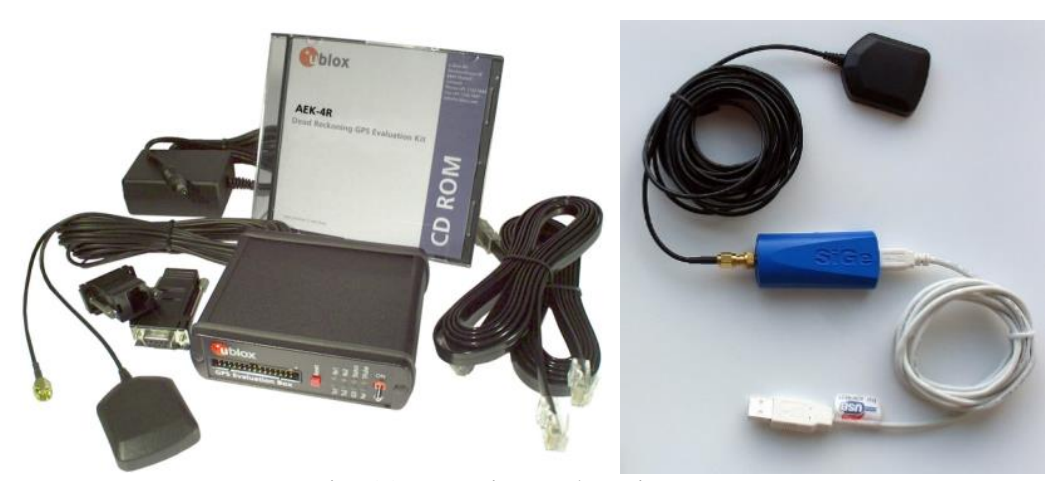

Fig. 11. Experimental equipment

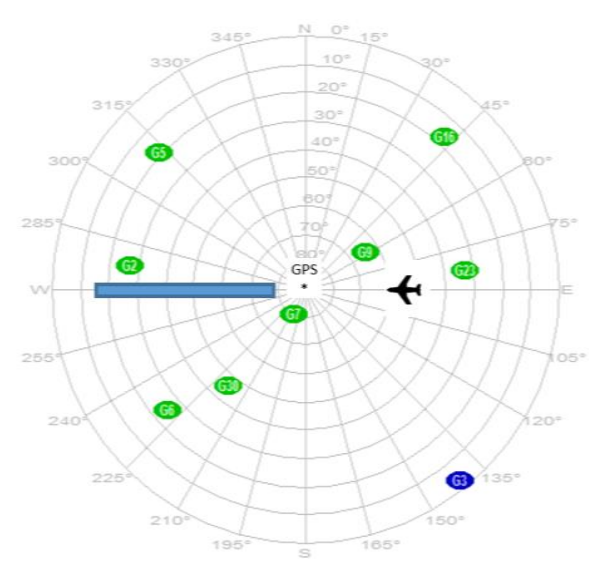

Fig. 12. Satellite constellation during the experiment

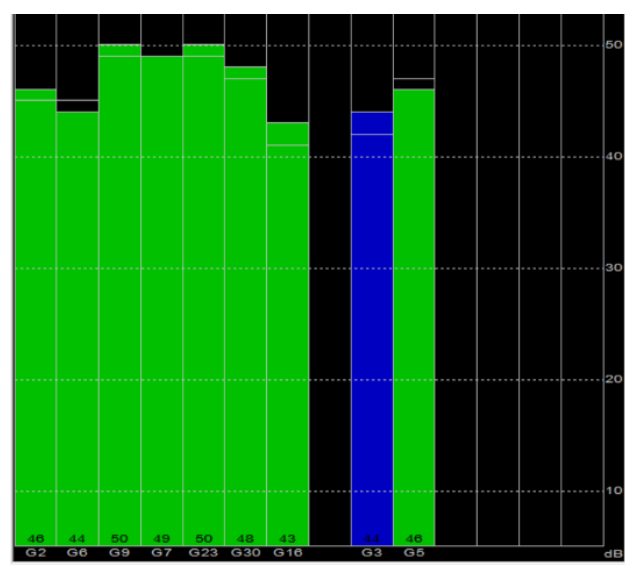

Fig. 13. Results of acquisition

During the experiment, the two GPS recording systems have been connected to the laptop for recording and storing the raw GPS data. The two recording systems have used the same GPS antenna mounted on the roof of a car that was stopped in $900 \mathrm{~m}$ from the start of the runway of the Sofia airport (Fig. 14).

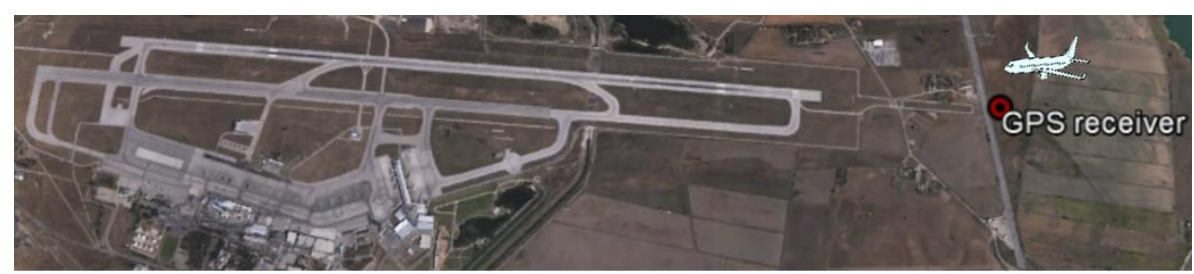

Fig. 14. Experiment topology 
In Fig. 14 is also shown the position of the receiver relative to the airport (the east-on the left, and the west-on the right). During the experiment, the GPS signal is recorded only at the moment when airplanes, taking off in the direction of the east or arriving from the east, are passed above the GPS receiver. Because the distance to the airplane is very small (about $100 \mathrm{~m}$ ), we expected that when the airplane crosses the baseline "satellite-receiver" or flies close to this line, the GPS signal is fully blocked by the airplane and the radio shadow (falling of the received signal) will be observed as a deep "hole" in the received signal. In our case the airplane will be located in the near zone of diffraction (geometric shadow). In that zone the distance to the target meets the inequality $R<<D^{2} / \lambda$.

Within 15-20 min were carried out two experiments. Under the conditions of the experiment there was no difference. The receiver was situated almost in the same place. A little difference was in the position of satellites, but also in the type and trajectory of landing airplanes, it did not affect the results of the experiment. For that reason, only the results of the first experiment are described here. As shown in Fig. 12, during the first experiment all visible satellites found by the GPS receiver "Antaris AEK-4R", have the following numbers: 2, 5, 6, 7, 9, 16, 23 and 30. These eight satellites and the GPS receiver together form the multi-channel radar system with eight transmitters and one common receiver. The airplane by crossing the virtual line "satellite-receiver" or by flying close to this line blocks the signals from some satellites and creates in that way the radio shadows in the corresponding FS system "satellites-receiver". The recorded GPS signal has been performed according to the algorithm shown in Fig. 6. The output signals of IF processing obtained in satellite channels of all visible satellites show that the signals of only satellites with numbers 2, 5 and 7 have been blocked by the airplane. The radio shadows, obtained in result of IF processing of the GPS signal, in satellite channels 2, 5 and 7 are shown in Figs 15-17.

However, the signals from the rest satellites $(6,9,16,23$ and 30) are not blocked by the airplane. For comparison the output of IF processing in the satellite channel 9 is shown in Fig. 18. It can be seen that the signal from Satellite 9 is not blocked by the airplane, and the radio shadow is not created.
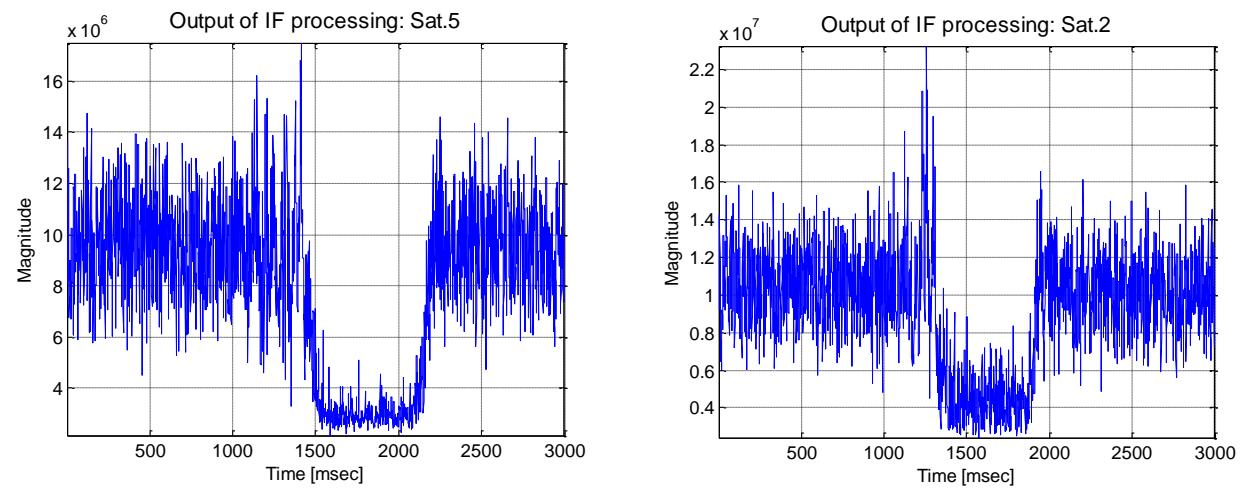

Fig. 15. Output signal of IF processing (Satellite 5) Fig. 16. Output signal of IF processing (Satellite 2) 

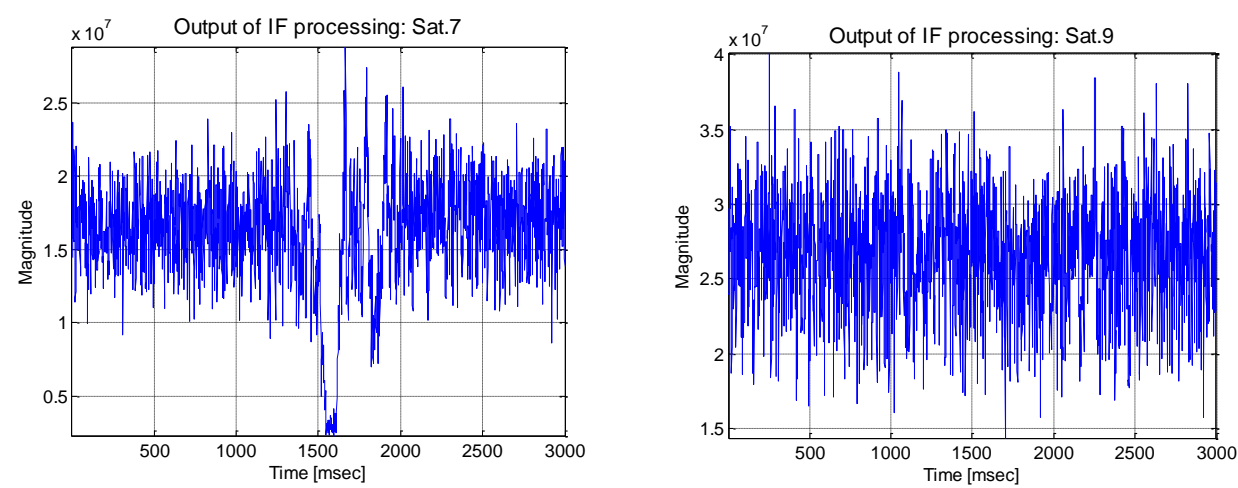

Fig. 17. Output signal of IF processing (Satellite 7) Fig. 18. Output signal of IF processing (Satellite 9)

Next, in each satellite channel, the outputs of the IF processing are inverted and are filtered in MAF before CFAR detection according to Fig. 2. Different criteria can be used for selection of the parameter $N$ in (8). For example, the parameter $N$ can be chosen in order to maximize SNR at the MAF output. Two variants of the parameter $N$ are calculated: 33 and 123. In this study we used the value $N=123$. The corresponding results of CFAR detection, obtained in channels 5, 2, 7, and 9 are shown in Figs 19, 20, 21 and together CFAR thresholds. It can be seen that the radio shadow created by the airplane is detected with high probability (SNR>16 dB) in three radar systems that are from Satellites 5, 2 and 7. In satellite channel 9, however, there is no detection, which means that the 9-th channel "Satellite 9-receiver "of the radar system cannot detect the air plane (Fig. 22). The probability of false alarm used to find the detection threshold is 0.001 .

The obtained results show that the target can be detected on his radio shadow with very high probability without a prior knowledge about what satellites are visible at the moment and where they are located. The more satellites detect the target, the higher the probability of target detection and fewer false alarms. In our case we used the OR logic in order to combine information about detections from all satellite channels. In order to decrease the probability of false alarm, the OR-logic can be replaced by the logic "K-of-M".

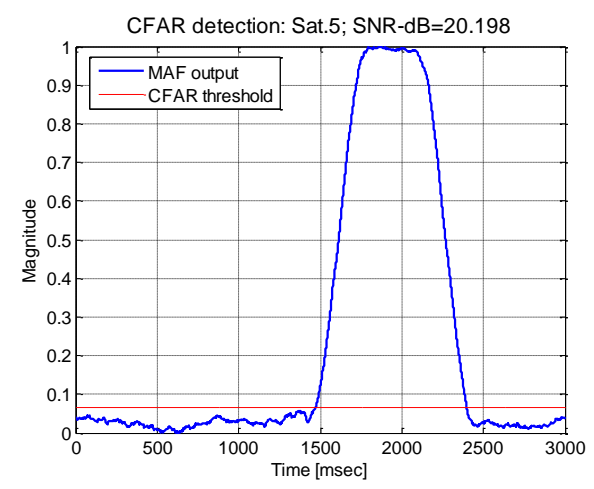

Fig. 19. CFAR detection (Satellite 5)

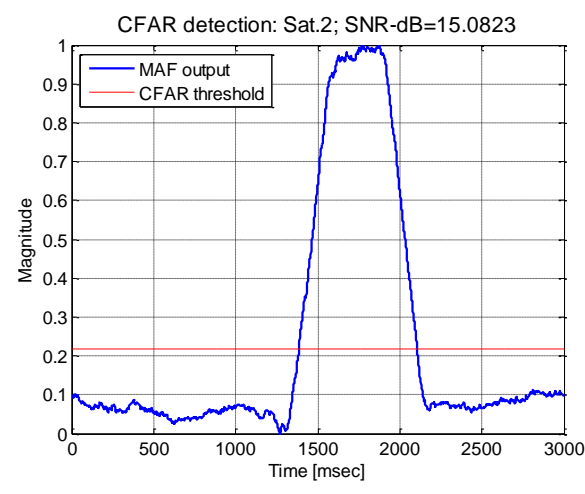

Fig. 20. CFAR detection (Satellite 2) 


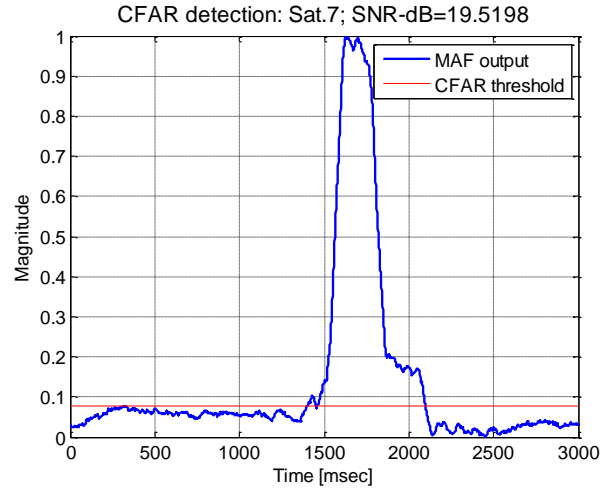

Fig. 21. CFAR detection (Satellite 7)

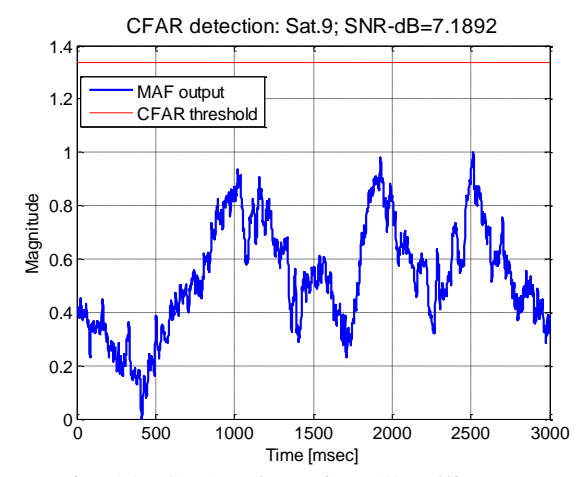

Fig. 22. CFAR detection (Satellite 9)

\section{Conclusion}

The results obtained in simulation in the far zone of diffraction and the experimental results obtained in the near zone of diffraction (geometric shadow) shows that it is possible to detect air objects on their GPS radio shadows by using of a multi-channel GPS FSR system when these objects cannot be detected by a bistatic radar system. The described multi-channel GPS FRS system exploits visible GPS satellites and one common receiver with a small omnidirectional commercial GPS antenna. Two experiments have been carried out close to the airport Sofia in order to test experimentally the proposed algorithm. The airplane is automatically detected in the multi-channel GPS FSR radar system if at least one of the processing channels registrate its detection.

Acknowledgements: This work was supported by Bulgarian Science Foundation, project DFNI-T 02/3/12.12.2014.

\section{References}

1. M. Cherniakov, Ed. Bistatic Radar: Principles and Practice. Wiley \& Sons, 2007.

2. Gle n n o n, E. P., A. G. D e m p s t e r, C. R i z o s. Feasibility of Air Target Detection Using GPS as a Bistatic Radar. - Journal of Global Positioning Systems, Vol. 5, 2006, No 1-2, pp. 119-126.

3. Cla ri zi a, M. P., P. B r a c a, C. Ruf, P. Willet t. Target Detection Using GPS Signals of Opportunity. - In: Proc. of 18th International Conference on Information Fusion, 2015, pp. 1429-1436.

4. Ko ch, V., R. We st ph a l. New Approach to a Multistatic Passive Radar Sensor for Airspace Defense. - IEEE AES Systems Magazine, 1995, pp. 24-32.

5. Suberviola, I., I. M a y ordome, J. Mendizabal. Experimental Results of Air Target Detection with GPS forward Scattering Radar. - IEEE Geoscience and Remote Sensing Letters, Vol. 9, 2012, No 1, pp. 47-51.

6. B o r r e, K., D. A k o s, N. B e r t e l s e n, P. R i n d e r, S. J e n s e n. A Software-Defined GPS and Galileo Receiver. Single-Frequency Approach. Boston, MA, Birkhäuser, 2006.

7. B e h a r, V., C. K a b a k c h i e v, H. R o h l in g. Air Target Detection Using Navigation Receivers Based on GPS L5 Signals. ION GNSS. Portland OR, 2011, pp. 333-337. 
8. G a r v a n o v, I., C. K a b a k c h i e v. Adaptive Binary Integration CFAR Processing for Secondary Surveillance. - Cybernetics and Information Technologies, Vol. 9, 2009, No 1, pp. 46-54.

9. Kabakchiev, C., I. Garvanov, V. B ehar, D. Kabakchieva, K. Kabakchiev, H. R o h 1 i n g, K. K u l p a, A. Y a r o v o y. Separation of GPS Signals in FSR System. - In: Proc. of 8th Microwave and Radar Week (MRW'18), 14-17 May 2018, Poznań, Poland, pp. 248-252.

10. K a b a k ch i e v, C., V. B e h a r, I. G a rv a n o v, D. K a b a k ch i e va, H. R o h 1 in g. Detection, Parametric Imaging and Classification of Very Small Marine Targets Emerged in Heavy Sea Clutter Utilizing GPS-Based forward Scattering Radar. - In: Proc. of 39th International Conference on Acoustics, Speech, and Signal Processing, Florence, Italy, 2014, pp. 793-797.

11. D a vid, A., C. B rou s s e a u, A. B o urdillo n. Simulations and Measurements of a Radar Cross Section of a Boeing 747-200 in the 20-60 MHz Frequency Band. - Radio Science, Vol. 38, 2003, Issue 4.

Received: 28.05.2018; Second Version: 05.12.2018; Accepted: 27.12.2018 